\title{
Second-order, high-temperature superconducting gradiometer for magnetocardiography in unshielded environment
}

\author{
Y. Zhang, ${ }^{\text {a) }}$ G. Panaitov, S. G. Wang, ${ }^{\text {b) }}$ N. Wolters, R. Otto, J. Schubert, W. Zander, \\ H.-J. Krause, H. Soltner, H. Bousack, and A. I. Braginskic) \\ Institut für Schicht- und Ionentechnik (ISI), Forschungszentrum Jülich (FZJ), D 52425 Jülich, Germany
}

(Received 21 September 1999; accepted for publication 16 December 1999)

\begin{abstract}
By employing high-temperature superconducting quantum interference device (SQUID) magnetometers, we have assembled a second-order gradiometer for magnetocardiography (MCG) in unshielded environment. With this high-temperature superconductor (HTS) SQUID system, we demonstrated its diagnostic relevance for MCG in terms of signal-to-noise ratio, spatial resolution, frequency bandwidth, rejection of environmental disturbances, and long-term stability. The electronically balanced gradiometer consists of three HTS radio-frequency SQUIDs with superconducting coplanar resonators, mounted in axial gradiometric arrangement with a baseline of $7.5 \mathrm{~cm}$. The system achieves a common mode rejection for axial homogeneous fields of about $10^{4}$ without any mechanical balancing, and a white noise about $130 \mathrm{fT} / \sqrt{ } \mathrm{Hz}$ at $77 \mathrm{~K}$, with an $8 \times 8 \mathrm{~mm}^{2}$ flux pickup area. MCG maps above volunteers' chests have been recorded in unshielded environment in a bandwidth of about $130 \mathrm{~Hz}$. We showed the influence of several notch filters (suppressing the power line frequency) on the quality of the MCG signals. (c) 2000 American Institute of Physics. [S0003-6951(00)03307-6]
\end{abstract}

Magnetocardiographic (MCG) measurements using superconducting quantum interference device (SQUID) sensors are usually performed in magnetically shielded rooms, to reduce the influence of electromagnetic disturbances from the environment. ${ }^{1,2}$ However, the high cost of such a room represents a major economic obstacle for the widespread application of magnetocardiography. Therefore, MCG gradiometer systems operating in unshielded environment would be highly desirable.

High-temperature superconductor (HTS) SQUID gradiometers of adequate sensitivity (magnetic field resolution) and good disturbance rejection could offer a lower system and operating cost than their low-temperature (LTS) equivalents. Until present, first- and second-order HTS SQUID gradiometers, measuring axial or tangential fields, were demonstrated and MCG data recorded without magnetic shielding. ${ }^{3-5}$ In these measurements, low-pass filters with a cutoff frequency of below $30 \mathrm{~Hz}$ were used to reduce the power line interference at $50 \mathrm{~Hz}$, or a $60 \mathrm{~Hz}$ notch filter was used. $^{5}$ However, the frequency components of the MCG higher than 50 and $60 \mathrm{~Hz}$ contain significant information., ${ }^{2,6}$ For clinical diagnostics, a MCG system has to have a bandwidth wider than $100 \mathrm{~Hz}$, so that further development is warranted. Electrocardiographic (ECG) instruments have bandwidths of $100 \mathrm{~Hz}$ or more.

In addition, a diagnostically meaningful map of magnetic field above the patient's chest has to be constructed of many sensing points (e.g., arranged in a $6 \times 6$ grid). Hence, a sufficient long-term stability of measurement is required if

\footnotetext{
${ }^{a}$ Electronic mail: y.zhang@fz-juelich.de

b) Permanent address: Department of Physics, Peking University, Beijing 100871, China.

c) Temporary address: Research Institute of Electrical Communication, Tohoku University, Sendai 980-8577, Japan.
}

sequential mapping is necessary. This is especially critical when using a single measuring point system. In this case, mapping can take, typically, about 40 min.

In this letter, we present an improved second-order HTS SQUID gradiometer addressing the requirements listed above. To demonstrate the diagnostic relevance of this system, $6 \times 6$ point field maps of healthy subjects were then recorded in unshielded environment.

We developed earlier HTS radio-frequency (rf) SQUIDs integrated with HTS coplanar resonators on $1 \mathrm{~cm}^{2}$ substrates. Their field resolution can be less than $B_{N}=30 \mathrm{fT} / \sqrt{ } \mathrm{Hz}$ at liquid nitrogen temperature, virtually adequate for diagnostic MCG. $^{7}$ On the basis of these SQUIDs, we constructed our second-order gradiometer (SOG). Below, we shall focus on the SQUID system technique related to MCG measurements.

Several SQUID magnetometers were first characterized in terms of flux noise inside a magnetically shielded cylinder. Of these, three SQUIDs of very similar performance were selected. Their average characteristics are listed in Table I. These SQUIDs were inserted in a sample holder in an axial arrangement at baseline distances of $7.5 \mathrm{~cm}$, to form a symmetric electronic gradiometer of second order.

TABLE I. Average characteristics of SQUID magnetometers with integrated coplanar resonators in a magnetic shielding.

\begin{tabular}{lc}
\hline \hline \multicolumn{1}{c}{ Parameter } & Value \\
\hline SQUID hole area $\left(\mu \mathrm{m}^{2}\right)$ & $150 \times 150$ \\
SQUID inductance $(p \mathrm{H})$ & 225 \\
Flux concentrator area $\left(\mathrm{mm}^{2}\right)$ & $8 \times 8$ \\
Pumping frequency $(\mathrm{MHz})$ & 830 \\
Field/flux coefficient, $\partial B / \partial \Phi\left(\mathrm{nT} / \Phi_{0}\right)$ & 2.5 \\
White field noise $(\mathrm{fT} / \sqrt{\mathrm{Hz}})$ & 40 \\
Slew rate $(\mathrm{mT} / \mathrm{s})$ & 1.3 \\
Dynamic range $(\mathrm{mT})$ & $> \pm 1$ \\
Total harmonic distortion $(\mathrm{THD})$ & $<10^{-5}$ \\
\hline \hline
\end{tabular}




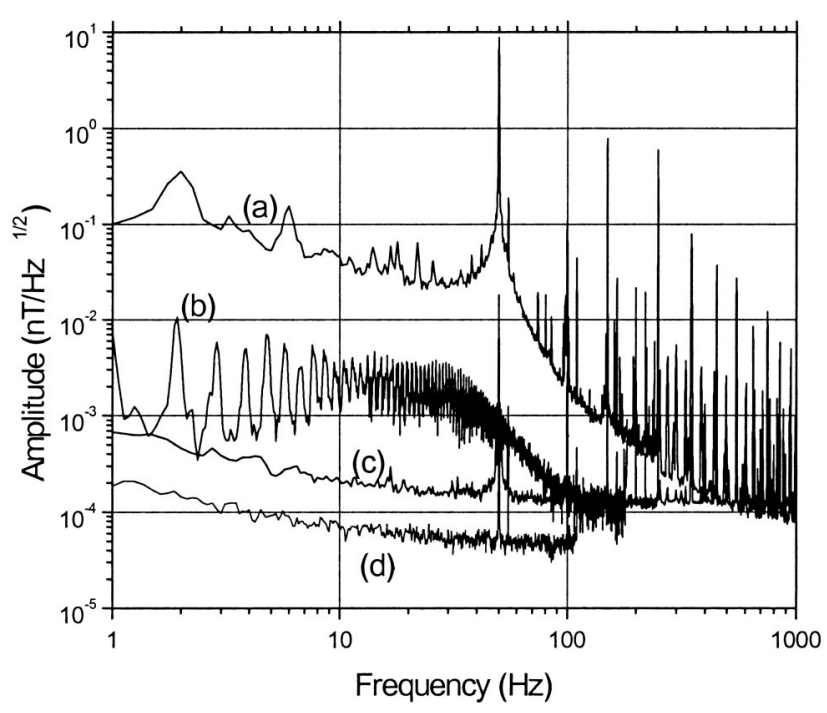

FIG. 1. Field spectra of (a) background noise measured by the magnetometer; (b) the magnetic signal of a healthy human heart; (c) the background after suppression by the second-order gradiometer in a laboratory without magnetic shielding. The noise of the magnetometer in shielding (d) is shown for comparison.

Figure 1 shows various magnetic spectra recorded with the magnetometer (one SQUID), and SOG. Trace (a) in Fig. 1 shows the spectral density of the background magnetic field disturbance measured by the magnetometer in our laboratory. It is seen that the disturbance is much higher than the spectrum of the heart signal shown as trace (b), even if the signal of the power line is not taken into account.

In trace (b), one sees that the heart signal is strongest at frequencies below $40 \mathrm{~Hz}$. As the frequency increases to 100 $\mathrm{Hz}$, the spectral density drops rapidly, down to the SOG white noise level of $130 \mathrm{fT} /{ } \mathrm{Hz}$ shown in trace (c). For different patients, the maximum amplitudes of MCG spectra may shift to higher or lower frequencies.

Trace (d) is the noise of the magnetometer measured inside magnetic shielding. Electronic combination of outputs of the three magnetometers, $\mathrm{A}, \mathrm{B}$, and $\mathrm{C}$, gives the $\mathrm{SOG}$ output $(\mathrm{A}-2 \mathrm{~B}+\mathrm{C})$. According to the theory of noise summation, the total noise of the SOG should be $\sqrt{6}$ times the noise of a single magnetometer in shielding, if the noise of the three SQUIDs is identical. ${ }^{8}$ This value can be regarded as the ultimate goal for an optimized gradiometer in an unshielded environment. By comparing trace (c) with trace (d), one sees that the performance of the SOG is close to this expected limit. Without shielding, the noise of each of the three magnetometers has increased above the spectrum (d), but not dramatically.

We performed electronic balancing by adjusting the relative output amplitudes of the three magnetometers via potentiometers (before subtraction). The common mode rejection (CMR) of a homogeneous axial field was measured to be about $10^{4}$ in a pair of Helmholtz coils with a diagonal of 2.8 $\mathrm{m}$, without any auxiliary mechanical adjustment. The optimum balance obtained in a homogeneous field may not always result in the lowest noise for the system in unshielded environment. Therefore, we adjusted the system to minimum noise at low frequencies, as shown in trace (c) of Fig. 1, despite the worse CMR values of only about $10^{3}$. This adaptive adjustment procedure was carried out at the measure-

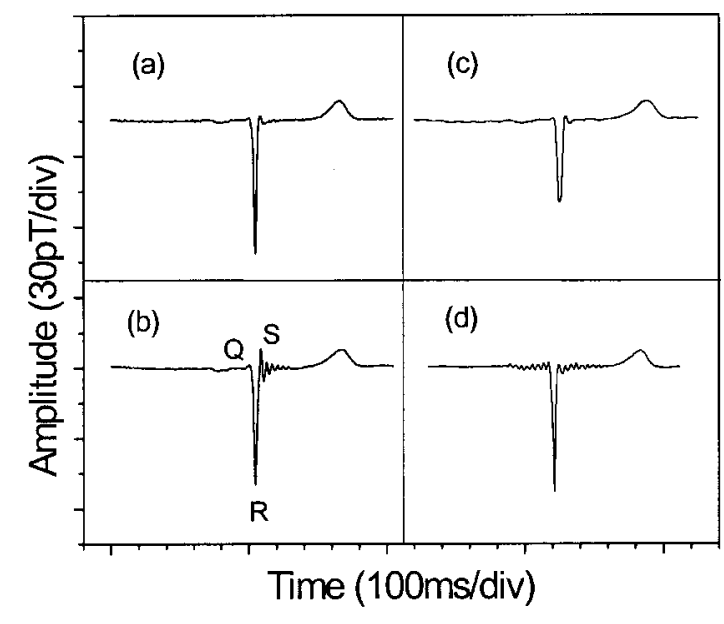

FIG. 2. MCG signals measured without and with $50 \mathrm{~Hz}$ notch filters, to show their influence. Trace (a) measured without $50 \mathrm{~Hz}$ notch filter; (b) with a notch filter of quality factor $Q=5$; (c) with a $Q=1$ notch filter; and (d) with a digital notch filter of $Q \approx 8$.

ment location and fixed position of the instrument, to account for the given disturbance environment.

We found that, in our laboratory, the peak-to-peak value of $25 \mathrm{nT}$ of the power line frequency $(50 \mathrm{~Hz})$ in the output of both magnetometers was reduced to $520 \mathrm{pT}$ in the output of the first order gradiometer (FOG), i.e., by about a factor of 50. This is still much higher than the typical peak value of the heart signal, about 100 pT. By adaptively optimizing the SOG, the peak-to-peak value of the $50 \mathrm{~Hz}$ source could be further reduced to about $4 \mathrm{pT}$. The output of the SOG was then dominated by $150 \mathrm{~Hz}$ disturbances. This frequency is higher than the upper bound of the heart signal spectrum, as seen in trace (b) of Fig. 1, and can be easily filtered out. A low-pass filter with a cutoff frequency of $130 \mathrm{~Hz}$ was employed to suppress the disturbances of the third and higher harmonics of $50 \mathrm{~Hz}$.

The $50 \mathrm{~Hz}$ component, however, is inside the frequency band of the heart signal. If the $50 \mathrm{~Hz}$ amplitude in the output of the gradiometer is still too high, the application of the 50 $\mathrm{Hz}$ notch filter could reduce the interference, but it causes a distortion of the signal. The main adverse influence of such a filter is an oscillation after the QRS complex in the MCG trace. The effect of notch filters with different quality factors $Q$ is shown in Fig. 2. In comparison with the MCG signal measured without any $50 \mathrm{~Hz}$ notch filter [Fig. 2(a)], a high $Q$ notch filter results in a comparatively low loss of the signal component, but it leads to a pronounced oscillation [Fig. 2(b)]. When the quality factor is reduced, the oscillation disappears, but the contribution of components in the vicinity of $50 \mathrm{~Hz}$ is also suppressed. One sees that the peak of the QRS complex is reduced to about $60 \%$ [Fig. 2(c)]. We also studied digital filtering via software with high $Q$ values. This resulted in oscillations before and after the QRS complex [Fig. 2(d)]. This effect may be due to time delay. The filtering experiment confirmed that the $50 \mathrm{~Hz}$ notch filter should be avoided to prevent the distortion of the measured data. Indeed, ECG instruments usually operate without a $50 \mathrm{~Hz}$ notch filter.

The real-time MCG traces of two persons, measured without the $50 \mathrm{~Hz}$ notch filter in a frequency bandwidth of about $130 \mathrm{~Hz}$, are shown in Fig. 3. The signal-to-noised ratio AIP license or copyright, see http://apl.aip.org/apl/copyright.jsp 


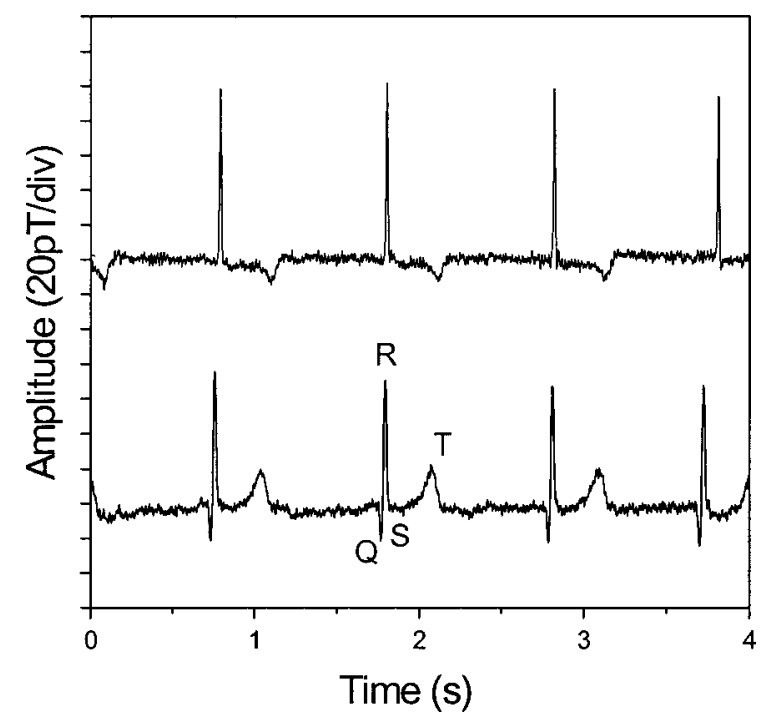

FIG. 3. Real-time magnetic signal of the heart of two different healthy persons, measured with a $130 \mathrm{~Hz}$ low pass and a $100 \mathrm{~Hz}$ notch filter. No 50 $\mathrm{Hz}$ notch filter was used in the measurements.

$(\mathrm{S} / \mathrm{N})$ is about 25 . Not only the the QRS complex, but also the $\mathrm{T}$ wave can be clearly seen in the real-time signal. Unfortunately, this signal-to-noise ratio is still not sufficient for medical analysis, so that in diagnostic measurements some averaging would be necessary. A lower noise floor, of $30 \mathrm{fT}$ or less, would make averaging less mandatory.

To demonstrate the potential of the system for clinical use, a 36-point mapping above the chest $(6 \times 6$ rectangular grid with spacing of $4.0 \mathrm{~cm}$ ) was carried out. The scanning over the 36-point map took about 40 min for our single sensor. Over that time period, the system provided a stable output without any reset or readjustment. Throughout the whole MCG mapping time, the system reduced the $50 \mathrm{~Hz}$ disturbance to a few pT. To increase the signal-to-noise ratio, we performed averaging while triggering with the ECG signal, which was recorded simultaneously. Figure 4 demonstrates a time-domain map of the heart signals. The traces were obtained by averaging 50 times. The signal-to-noise ratio is about 300 at point E4. Such averaged traces can form the necessary data base for medical analysis.

To summarize, we have developed an electronic secondorder axial HTS SQUID gradiometer, which enables us to perform MCG measurements without magnetic shielding in a frequency band up to $130 \mathrm{~Hz}$. It has a white noise of 130 $\mathrm{fT} / \sqrt{\mathrm{Hz}}$, and a high spatial resolution due to the small size of the SQUIDs. For the first time, a 36-point MCG mapping has

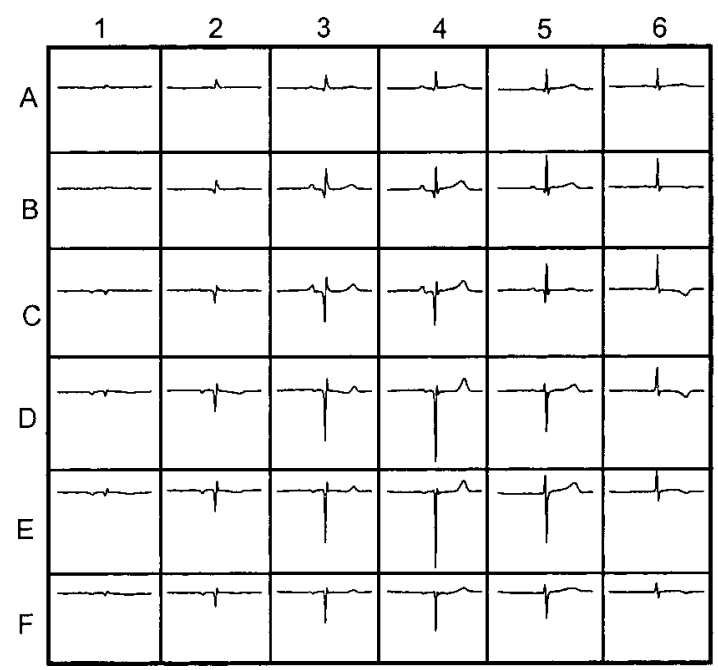

FIG. 4. 6×6-point MCG mapping above the chest of a normal subject. The data were obtained without $50 \mathrm{~Hz}$ notch filter by averaging 50 heartbeats.

been recorded by using an HTS SQUID gradiometer in an unshielded environment. This demonstrates the relevance of the gradiometer for MCG diagnostics. Future work should aim at an effective solution for third-order gradiometry needed in very noisy hospital environments, and suitable for multichannel systems. One should also aim at still lower SQUID noise floor (higher $\mathrm{S} / \mathrm{N}$ ), and reduction of $1 / f$ noise. Ultimate multichannel MCG systems will then ensue.

The authors would like to thank E. Zimmerman for assistance with digital filters. This work was supported by the German BMBF under Contract No. 13 N 7327.

${ }^{1}$ P. J. Karp, T. E. Katila, M. Saarinen, P. Siltanen, and T. T. Varpula, Circ. Res. 47, 117 (1980)

${ }^{2}$ S. N. Erné, R. R. Fenici, H.-D. Hahlbohm, J. Korsukewitz, H. P. Lehmann, and Y. Uchikawa, in Biomagnetism: Application and Theory, edited by H. Weinberg, G. Stroink, and T. Katila (Pergamon, New York, 1984), p. 132.

${ }^{3}$ Y. Tavrin, T. Zhang, M. Mück, A. I. Braginski, and C. Heiden, Appl. Phys. Lett. 62, 1824 (1993).

${ }^{4}$ J. Borgmann, P. David, G. Ockenfuss, R. Otto, J. Schubert, W. Zander, and A. I. Braginski, Rev. Sci. Instrum. 68, 2730 (1997).

${ }^{5}$ K. A. Kouznetsov, J. Borgmann, and J. Clarke, Appl. Phys. Lett. 75, 1979 (1999).

${ }^{6}$ P. Endt, H.-D. Hahlbohm, D. Kreiseler, M. Oeff, U. Steinhoff, and L. Trahms, Med. Biol. Eng. Comput. 36, 723 (1998).

${ }^{7}$ Y. Zhang, N. Wolters, X. H. Zeng, J. Schubert, W. Zander, H. Soltner, H. R. Yi, M. Banzet, F. Rüders, and A. I. Braginski, Appl. Supercond. 6, 385 (1998).

${ }^{8}$ J. Vrba, in SQUID Sensors: Fundamentals, Fabrication and Applications, NATO ASI Series, edited by H. Weinstock (Kluwer, Dordrecht, 1996), p. 117. 